\title{
A comparison of instruction formats for administering a computerized behavioral test
}

\author{
W. KENT ANGER, DIANE S. ROHLMAN, and O. J. SIZEMORE \\ Oregon Health Sciences University, Portland, Oregon
}

\begin{abstract}
Shaping and fading techniques were employed to replace written instructions used in computerized behavioral tests. Apple Macintosh computers and HyperCard with Pascal extensions were chosen as the development environment to implement the new techniques. Initial findings demonstrate the effectiveness and relative efficiency of shaping techniques to teach correct performance on the Symbol-Digit Test.
\end{abstract}

Behavioral tests are used to identify neurotoxic effects in human populations. These primarily neuropsychological tests discriminate between chemically exposed groups and reference groups not exposed to chemicals (Anger, 1990). The battery of tests most widely used in neurotoxicology research is the computerized Neurobehavioral Evaluation System (NES; Baker, Letz, \& Fidler, 1985; Letz, 1990), although traditional, personal test-administration techniques are also used widely (e.g., Anger, 1990; Anger et al., 1993).

The most serious limitation of the current tests used in neurotoxicology and other research areas is their dependence on written instructions. This feature limits their utility among subjects with poor language skills and requires translation to test people who speak different languages. These limitations can be reduced or eliminated by an instructional approach that employs shaping techniques to modify responding, and techniques such as fading to alter stimulus characteristics associated with responding.

Shaping procedures were early techniques used to teach simple discriminations to animals (e.g., Lashley, 1939). However, with the advent of operant techniques, responseshaping and stimulus-fading procedures have been successfully employed to teach a wide range of behaviors in a variety of species (e.g., Gleeson, 1991; Harrison, 1991; Sulzer-Azaroff \& Gillat, 1990; Terrace, 1966; Tudor \& Bostow, 1991). The training of complex behavior is achieved by dividing the task into a series of small steps and rewarding successive approximations until the subject has mastered the task. Thus, shaping and fading can replace language in behavioral testing by using a consistent set of operations that function without regard to cultural, language, or educational background. Shaping is used here as a generic descriptor to encompass the vari-

Preparation of this article was supported in part by Environmental Protection Agency Grant R820111-01-0 and National Institute of Environmental Health Sciences Grant RO1ESO6475-01 to W. K. Anger. We gratefully acknowledge the assistance of Julie Glasser and Joanne Brown with data collection and subject recruitment. Requests for reprints should be sent to W. K. Anger, CROET L606, Oregon Health Sciences University, 3181 SW Sam Jackson Park Road, Portland, OR 97201. ous shaping, fading, and modeling techniques employed in the instruction format reported in this article.

\section{Development Environment}

The initial approach to test development is the selection of an environment that allows response-shaping and stimulus-modification techniques to be used. The development environment has three components: the equipment platform, the software environment, and the user interface. Table 1 shows these components as well as the minimum requirements for each.

The Apple Macintosh platform was chosen for the development environment because it meets the basic requirements and has the capability of playing digitized video and animation clips on a personal computer screen through its QuickTime software.

HyperCard was chosen to meet the software and user interface requirements. Its level of complexity is intermediate between source code and fourth-generation languages, and thus provides a relatively short development time for the programmer. "Authoring"' allows the entrylevel programmer to create or modify program elements by using buttons, fields, and cards. However, HyperCard also includes a second level of programming, termed "HyperTalk" - an English-like scripting language-for the creation of highly complex applications. External subroutines written in source code languages can be incorporated into a HyperCard program to improve speed and performance. External subroutines can also add the capability of millisecond timing. Finally, HyperCard has a unique plug-and-play capacity that allows persons with very limited computer experience to readily utilize complex applications.

\section{A Prototype Test: The Symbol-Digit Test}

The Symbol-Digit Coding Test (Baker et al., 1985) is a widely used, computerized version of the Wechsler Adult Intelligence Scale (WAIS-R) Digit-Symbol Test (Wechsler, 1981). In this task, nine unique symbols are paired with the numbers 1-9. A matrix containing the symbols and blank spaces provides a workspace in which the subject can type the corresponding number of each 
Table 1

Requirements for Selecting a Development Environment

Platform Requirements

1. Worldwide availability

2. Portable computers

3. External device input

4. Audio/video capabilities

Software Requirements

1. Visual display control

2. Millisecond timing

3. Control of audio and video

4. Flexible file manipulation

User Interface Requirements

1. Plug and play capability

2. Economy of programming

3. Short learning curve

pair. This test requires the subject to (1) press a key when a number appears, (2) press the key quickly, (3) learn the symbol-digit relationship, and (4) fill in the matrix.

The first step in shaping performance on our prototype test, the Symbol-Digit Test, involves pressing a key when a number appears. In this study, this skill was trained by a "Shaping Tutorial"' in which the subject is first shown a drawing of a laptop (PowerBook) screen and keyboard used in this study with a drawing of a hand at the bottom. Shortly thereafter, a large number appears on the screen and the key with the same number becomes outlined on the pictured keyboard (Figure 1, panel A). If the subject does not press the key within a few seconds, animation is used to model the keypress (viz., the drawn hand moves up and appears to "press" the key on the screen). Once the key is pressed (either by the subject or by the drawn hand), the key becomes dark (providing response feedback) and the number disappears. If the subject presses the correct key, a large "smiling face" appears on the screen (Figure 1, panel B). This stimulus was selected because of the universal nature of facial expressions of emotions (Ekman \& Friesen, 1975). It is used throughout the test as a mildly reinforcing stimulus to signal "yes," "correct," or "good job." After the subject makes two correct keypresses, the picture of the PowerBook disappears and the keyboard image moves in a series of steps to the bottom of the screen. Two additional correct keypresses in response to number presentations are required to complete the Shaping Tutorial.

The first step in training performance on the SymbolDigit Test is to convey the concept of working rapidly. This is done by displaying an empty circle along with a number. The circle fills, simulating a clock face with a sweeping second hand, until a key is pressed (Figure 1, panel C). The speed at which the circle fills is increased on successive trials. If the circle fills before the subject presses the correct key, a negative stimulus (frowning face) appears, followed by a 2-sec time-out (TO) period (unavailability of responding on the task). The TO is also initiated if the subject presses an incorrect key (e.g., presses the 6 key when a 9 was presented on the screen). At the fastest speed, the circle is filled in $2.5 \mathrm{sec}$. The subject must complete two correct key responses before moving to the next shaping stage.

To demonstrate the relationship between the symbols and the digits, a single symbol-digit pair is presented. Below it, the symbol alone appears in a matrix outline (Figure 1, panel D), and the corresponding key is outlined on the keyboard image. If the subject presses the correct key, the smiling face appears. A fading procedure is used in the subsequent steps to gradually introduce the full matrix of symbol-digit pairs-that is, the numbers, the symbols, and the lines of the matrix change from faint, to gray, to black (Figure 1, panels E-H). As the subject progresses through these stages, the bottom matrix is successively filled from left to right.

\section{Performance on the Symbol-Digit Test}

To evaluate the new instruction format, the SymbolDigit Test was administered to three different groups of subjects using the shaping instructions and written instructions. Of interest was whether or not training time or performance would differ across instruction formats. The shaping instructions were as described above, and the Neurobehavioral Evaluation System (NES) computerized Symbol-Digit Test provided the comparable test with

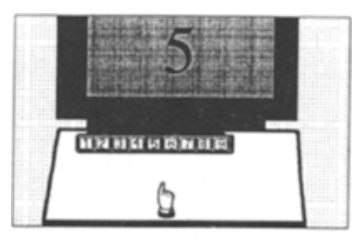

Panel A.

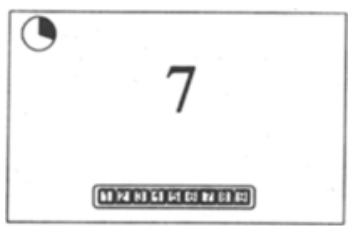

Panel C.

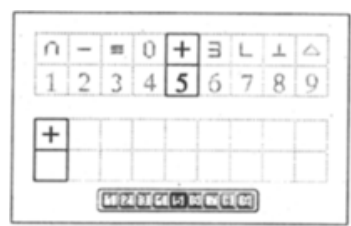

Panel E.

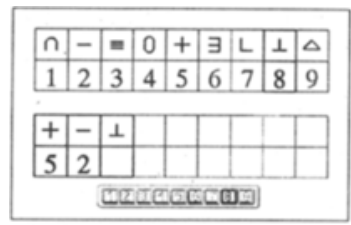

Panel G.

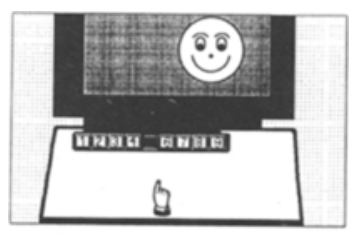

Panel B.

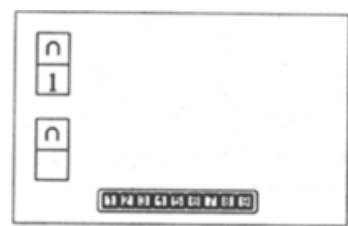

Panel D.

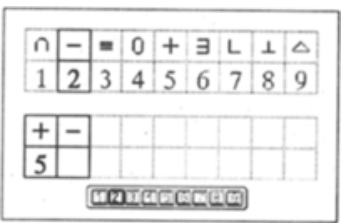

Panel F.

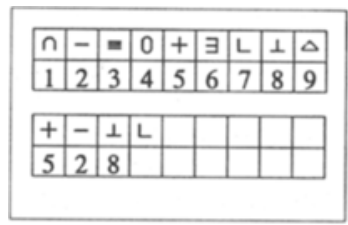

Panel H.
Figure 1. Shaping steps used to train performance on the SymbolDigit Test. The digit-symbol combinations from the Wechsler Adult Intelligence Scale were used in the study, but have been replaced in this figure due to copyright restrictions. 
written instructions. The effectiveness of the filling circle to convey the concept of "work as quickly as you can" in the shaping instructions also needed an empirical assessment. Thus, a version of the shaping instructions that did not include this feature was developed and administered to the third group of subjects. The NES written instructions included the words "go as fast as you can."

\section{METHOD}

\section{Subjects}

Forty-eight subjects were recruited from an advertisement in a campus publication at Oregon Health Sciences University (OHSU). Each was paid $\$ 20$ for completion of the Symbol-Digit Test and additional tests not described in this paper. Power analyses conducted with data from previous studies with the NES suggested that 16 subjects per cell were required to detect a difference equal to two thirds of the standard deviation with a power of $95 \%$ and an alpha level of .05 with a two-tailed test. The subjects' mean age varied between 29 and 32 years, and their mean years of education varied between 14 and 15 . The mean frequency of computer use was rated by these subjects as 4.5-4.7 on a scale of 1 (never used a computer) to 5 (use a computer almost daily; see Table 2).

\section{Procedure}

The Symbol-Digit Test with shaping instructions (both with and without speed training) was administered on a PowerBook 180 portable computer with a plastic overlay covering all of the keys except the number keys. The following instructions were read to the subjects: "The task you will perform today requires you to solve a problem. As a result I cannot answer any questions that might arise while you are working on the task." The Symbol-Digit Test with written instructions was administered on a Toshiba portable computer (Model 3200 or 5200 ) with a plastic overlay covering all of the keys except the number keys. These instructions were read to the subjects: "The instructions for this task have been entered into the computer and will appear on the screen. If you cannot read them or they are not clear, call me to assist you." None of the subjects asked any questions during the study.

All the subjects completed one practice trial and five test trials (symbol-digit matrices). They were allowed to make only one response for each symbol-digit pair in a trial. In other words, incorrect responses were accepted during test trials.

\section{RESULTS}

The data collected for each group include the amount of time required for training, the total amount of time required to complete the five test trials for the Symbol-Digit Test, and the latency for each response. Training time was measured from the start of the task until the beginning of the test trials. For the written instructions, this included

Table 2

Mean Age, Years of Education, and Frequency of Computer Use (Scale of 1-5) for Each Instruction Format

\begin{tabular}{|c|c|c|c|c|c|c|}
\hline \multirow[b]{2}{*}{ Instructions } & \multicolumn{2}{|c|}{ Age } & \multicolumn{2}{|c|}{$\begin{array}{l}\text { Years of } \\
\text { Education }\end{array}$} & \multicolumn{2}{|c|}{$\begin{array}{c}\text { Computer } \\
\text { Use }\end{array}$} \\
\hline & $M$ & $S D$ & $M$ & $\overline{S D}$ & $M$ & $S D$ \\
\hline Shaping (speed) & 29 & 5.4 & 15 & 2.1 & 4.6 & 0.9 \\
\hline Shaping (no speed) & 31 & 11.4 & 15 & 2.4 & 4.5 & 0.9 \\
\hline Written (NES) & 32 & 9.5 & 14 & 1.3 & 4.7 & 0.7 \\
\hline
\end{tabular}

the time required to read the various instructions and complete the practice trial. For the shaping instructions, this measure included the time required to teach the subject to press a number quickly, to learn to pair the symbols with numbers, and to complete the practice matrix. Following the training component, the latency was measured for each keypress by timing the period from the opportunity to respond (i.e., appearance of the matrix) until a response was emitted. Overall test performance was evaluated by combining the latency for each keypress. All times were measured to the nearest 10 th of a second.

The test session consisted of five test trials; each trial required nine responses. The symbol-digit pairings in both symbol-digit tests were random (i.e., on each trial a different number was paired with a different symbol). Although different symbols were used in the shaped and written versions, the sequence of correct responses was the same for all the subjects. Any errors made by the subjects during the test trials were also recorded.

Thirty of the 32 subjects in the two shaping conditions completed the test rapidly. Strict termination criteria severely limited practice time, and 2 of the subjects initially failed the task. However, these 2 subjects learned quickly when given the same test with shaping instructions 1 week later. Their data are not included in the following analyses.

A one-way analysis of variance showed no significant effect of instruction format on training time $[F(2,43)=$ $.76, p=.475]$. As seen in Figure 2, mean training time was virtually the same for the shaping instructions with speed training $(80 \mathrm{sec})$ and NES written instructions $(79 \mathrm{sec})$, whereas shaping instructions without speed training took slightly longer and had a great deal more variability (standard deviation of 55 vs. 9 and 14 for shaping with speed training and NES written instructions, respectively).

Further, the effect of instruction format approached but did not exceed the .05 significance level $[F(2,43)=3.02$, $p=.059]$. However, the subjects who had received shaping with speed training were $10.5 \mathrm{sec}$ faster (or about $10 \%$ ) in the test performance period than the subjects who had received NES written instructions (see Figure 2). On the other hand, the NES subjects were less variable (standard deviation of 7.4 vs. 14 for shaping with speed training and 23.5 for shaping without speed training).

The subjects made very few incorrect responses; the mean number of errors per subject in five trials was 0.54 . There were no statistically significant differences between the groups $[F(2,43)=2.27, p=.12]$.

\section{DISCUSSION}

Instruction format did not influence training time or test performance time on the Symbol-Digit Test when shaping instructions were compared with written instructions in a widely used computer-implemented battery. Although shaping training on this task required more time $(80-$ $92 \mathrm{sec}$ ) than did written instructions on the NES (79 sec), the difference has no practical significance. Shaping instructions appear to be as effective for training Symbol- 


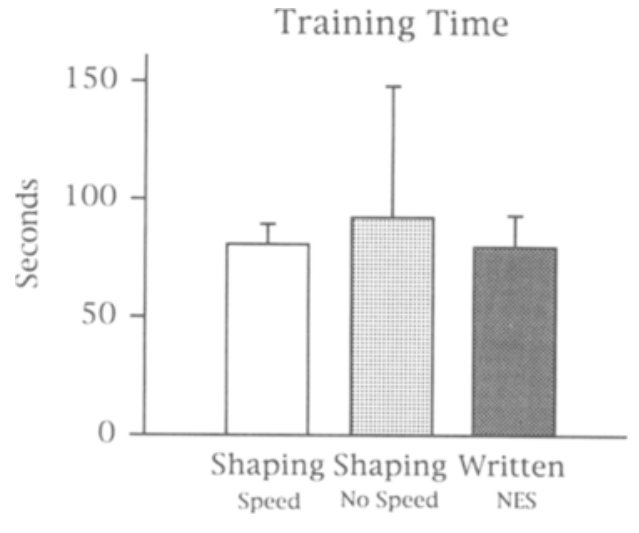

Test Performance Time

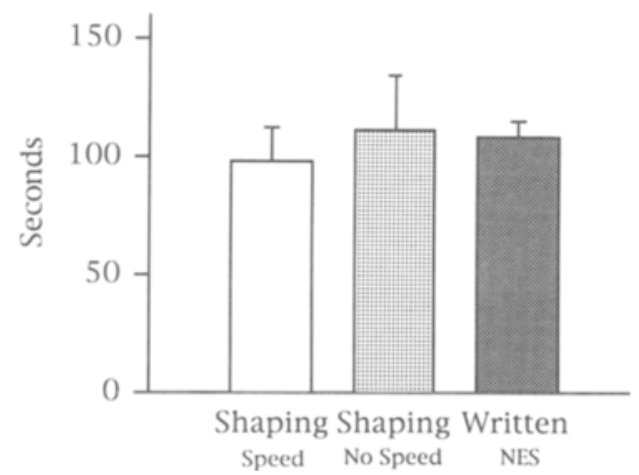

Figure 2. Mean training and test performance time (in seconds) for each instruction format.

Digit Test performance as written instructions, without adversely influencing test performance.

The filling circle conveyed the concept of "work as quickly as you can," as effectively as the NES written instructions. In fact, the subjects who were given shaping with speed training performed more rapidly than any other group, although the differences were not significant. The data also reveal that the group that took the longest time on the trials were the subjects who were given the shaping instructions without the speed training. Although their performance speed was not significantly different from the group that was given the shaping instructions with the speed training, their speed was slower and the difference approached significance $[t(28)=1.94, p=$ .06].

Shaping is an efficient method of training performance on the Symbol-Digit Task and thus offers a viable alternative training method that has obvious potential for efficiently testing people who are illiterate, have poor language skills, or do not speak English.

\section{REFERENCES}

ANGER, W. K. (1990). Worksite behavioral research: Results, sensitive methods, test batteries and the transition from laboratory data to human health. NeuroToxicology, 11, 629-720.

Anger, W. K., Cassitto, M. G., Liang, Y., Amador, R., Hooisma, J., Chrislip, D. W., Mergler, D., Keifer, M., HorTNAGL, J., Fournier, L., Dudek, B., \& Zsogon, E. (1993). Comparison of performance from three continents on the WHO-Recommended Neurobehavioral Core Test Battery. Environmental Research, 62, 125-147.

BAKER, E. L., LETZ, R., \& FidLer, A. (1985). A computer-administered neurobehavioral evaluation system for occupational and environmental epidemiology, Journal of Occupational Medicine, 27, 206-212.

EKMAN, P., \& FRIESEN, W. V. (1975). Unmasking the face. Englewood Cliffs, NJ: Prentice-Hall.

GleEson, S. (1991). Response acquisition. In I. H. Iverson \& K. A. Lattal (Eds.), Analysis of behavior: Part I. Techniques in the behavioral and neural sciences (pp. 63-86). New York: Elsevier.

Harrison, J. M. (1991). Stimulus control. In I. H. Iverson \& K. A. Lattal (Eds.), Analysis of behavior: Part I. Techniques in the behavioral and neural sciences (pp. 251-292). New York: Elsevier.

LASHLEY, K. S. (1939). The mechanism of vision: XVI. The functioning of small remnants of the visual cortex. Journal of Comparative Neurology, 70, 45-67.

LETz, R. (1990). The neurobehavioral evaluation system: An international effort. In B. L. Johnson, W. K. Anger, A. Durao, \& C. Xintaras (Eds.), Advances in neurobehavioral toxicology: Applications in environmental and occupational health (pp. 189-201). Chelsea, MI: Lewis.

Sulzer-Azaroff, B., \& Gillat, A. (1990). Trends in behavioral analysis in education. Journal of Applied Behavioral Analysis, 23, 491-495.

Terrace, H. S. (1966). Stimulus control. In W. K. Honig (Ed.), Operant behavior: Areas of research and application (pp. 271-344). New York: Appleton-Century-Crofts.

Tudor, R. M., \& Bostow, D. E. (1991). Computer-programmed instruction: The relation of required interaction to practical application. Journal of Applied Behavioral Analysis, 24, 361-368.

WeChSLER, D. (1981). WAIS-R manual. New York: Psychological Corporation. 